\title{
ON THE STRUCTURAL DESIGN OF IMPERFECTION SENSITIVE LAMINATED COMPOSITE SHELL STRUCTURES SUBJECTED TO AXIAL COMPRESSION
}

\author{
Linus Friedrich ${ }^{1}$, Pawel Lyssakow ${ }^{1,2}$, Garth Pearce $^{2}$, Martin Ruess $^{3}$, Chiara Bisagni ${ }^{4}$, \\ Kai-Uwe Schröder ${ }^{1}$
}

\author{
${ }^{1}$ Institute of Structural Mechanics and Lightweight Design, RTWH Aachen University \\ Wüllnerstrae 7, 52062 Aachen, Germany \\ e-mail: Linus.Friedrich@sla.rwth-aachen.de,Pawel.Lyssakow@rwth-aachen.de, \\ Kai-Uwe.Schroeder@sla.rwth-aachen.de \\ ${ }^{2}$ School of Mechanical and Manufacturing Engineering, UNSW Sydney \\ Sydney NSW 2052, Australia \\ e-mail: g.pearce@unsw.edu.au
${ }^{3}$ School of Engineering, University of Glasgow
Ranking Building, Oakfield Avenue Glasgow G12 8LT, United Kingdom e-mail: martin.ruess@ glasgow.ac.uk \\ ${ }^{4}$ Aerospace Structures and Computational Mechanics, Delft University of Technology \\ Kluyverweg 1, 2629HS Delft, The Netherlands \\ e-mail: C.Bisagni@tudelft.nl
}

Keywords: Shell buckling, imperfection sensitivity, composite shells.

\begin{abstract}
Shell structures are used as primary structures of space launch vehicles. These structures are thin-walled and are thus prone to buckling when loaded in compression. Because of the imperfection sensitivity of these structures, small deviations of the real shell from the theoretically perfect shell may result in a tremendous decrease in load carrying capacity. For this reason, geometrical imperfections need to be taken into account. When designing unstiffened composite shells, the laminate stacking sequence influences both, the buckling load of the geometrically perfect shell and the imperfection sensitivity of the shell. Consequently, to derive laminate stacking sequences that maximize the buckling load of real shell structures, geometrical imperfections need to be taken into account already in an early design phase. In this paper, two laminate stacking sequences that were derived to maximize the buckling load of the geometrically perfect and imperfect shell structure are studied using stochastic methods. To this end, combination of non-rotational symmetric imperfections derived from measured data and variations of the ply orientation are studied in a stochastic analysis on basis of Monte Carlo simulations. The results of this study will be used to evaluate the influence of the stacking sequence as one of the essential properties dominating the structural response of geometrically imperfect laminate composite shell structures.
\end{abstract}




\section{INTRODUCTION}

The structural design of imperfection sensitive shells is one of the problems constantly addressed in the field of mechanics. Due to the imperfection sensitivity of thin walled shells, deriving reliable design loads is a demanding task and it is mainly focused on how imperfections can be taken into account. To this end, several approaches were developed as, for example, summarized by Hoff [1] and Bushnell [2]. Recent approaches to account for geometrical imperfections are summarized by Castro [3] and Friedrich [4].

When designing imperfection sensitive isotropic shell structures, the design variable is the shell wall thickness and the shell's dimensions, which are defined on a system level. The load carrying capacity of laminated composite shell structures is additionally influenced by the laminate stacking sequence, see Figure 1 . The laminate stacking sequence influences:

- the buckling load of the geometrically perfect shell and the shell's imperfection sensitivity, which results in a theoretical buckling load prediction

- the imperfection pattern of a manufactured shell and thus, the real buckling load is influenced by the shell's laminate stacking sequence

Consequently, it can be stated that the optimization of a laminate stacking sequence for a shell structure with regard to the load carrying capacity of the real shell is associated with many uncertainties. This drawback eliminates the possibility of performing structural optimizations, especially in an early structural design phase as such optimizations are time consuming and computationally costly and do not necessarily yield useful results because of the high number of uncertainties involved.

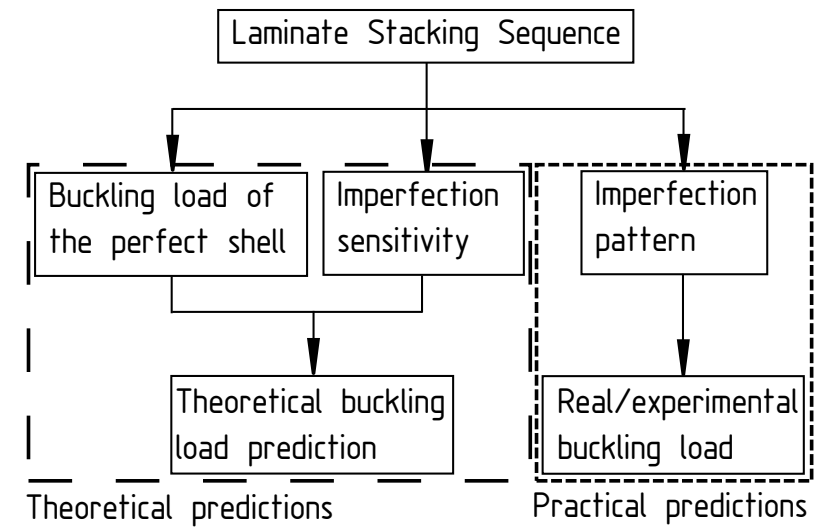

Figure 1: Influence of the laminate stacking sequence of composite shell structures on the structural response

Due to the high number of uncertainties, simplifications with regard to the imperfection pattern and boundary conditions need to be accepted to maximize the buckling load of imperfection sensitive composite shell structures. Based on these simplifications laminate stacking sequences can be identified, which are thought to maximize the buckling load of real shell structures. Shell structures derived based on two different approaches are further studied in this paper. One, a shell structure identified by Zimmermann in the 1990s [5] and two, a shell structure recently identified by Friedrich et. al. [6]. This paper is structured as follows: the study structures taken into account are introduced in section two. Subsequently, results determined using stochastic analyses are presented in section three and the paper closes with a summary and outlook in section four. 
Table 1: Overview of material properties [5]

\begin{tabular}{cr}
\hline \hline Properties & Composite shells \\
\hline $\mathrm{E}_{11}$ & $123551 \mathrm{MPa}$ \\
$\mathrm{E}_{22}$ & $8708 \mathrm{MPa}$ \\
$\mathrm{G}_{12}$ & $5695 \mathrm{MPa}$ \\
$\nu_{12}$ & 0.32 \\
\hline \hline
\end{tabular}

\section{INTRODUCTION OF STUDY STRUCTURES}

Two different approaches to derive the study structures here discussed were taken into account. One, the laminate stacking sequence were derived to maximize the buckling load of geometrically perfect shell structures. The shell structures have been derived by Zimmermann in the 1990s [5, 7] and the resulting shells were denoted Z-shells. Two, the laminate stacking sequence were derived to maximize the buckling load of geometrically imperfect shells with rotational symmetric imperfections. The shell structures have been derived by Friedrich et. al. [6] and the resulting shells were denoted FL-shells.

The material properties used in Zimmermann [5] and Friedrich [6] are summarized in Table 1. The geometrical properties are defined as follows: the inner radius of the shell was 250 $\mathrm{mm}$ and the length of the shell was $510 \mathrm{~mm}$. The length to radius ratio is about two and thus, the influence of the length of the shell and the type of boundary conditions do not affect the buckling load of the shell structure as shown in [8].

The results of deriving laminate stacking sequences that maximize the buckling load of a geometrically perfect and geometrically imperfect shells are summarized in Table 2 for those shells with two and five angle plies, that is $n_{p}=2$ and $n_{p}=5$. Additionally, the laminate stacking sequences influence on the buckling load of the geometrically perfect and geometrically imperfect shell with rotational symmetric imperfections are given in Figures 2 and 3 for two angle plies and an imperfection magnitude of 0.2 times the shell wall thickness that is $\mathrm{w}_{0} / t=0.2$. The buckling loads of the $n_{p}=2$-shell are presented in detail since the buckling loads could be represented with reference to the ply orientation of the inner and outer ply and this allowed a suitable graphical evaluation of results.

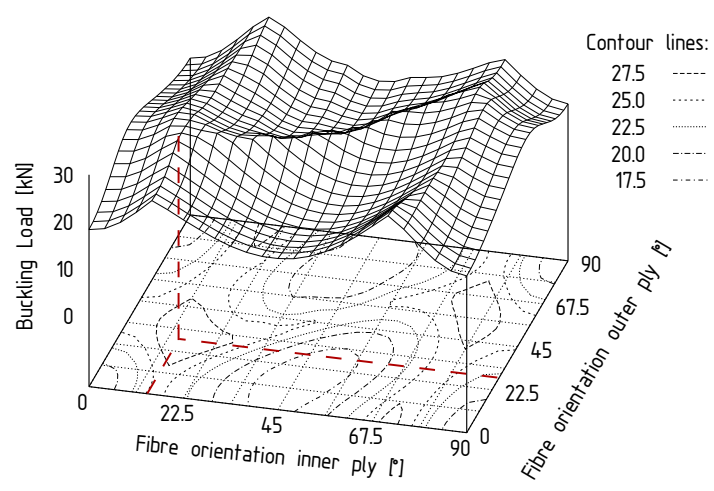

Figure 2: Buckling load of the perfect shell structure $\mathrm{n}_{\mathrm{p}}=2$ according to Friedrich $[6]$

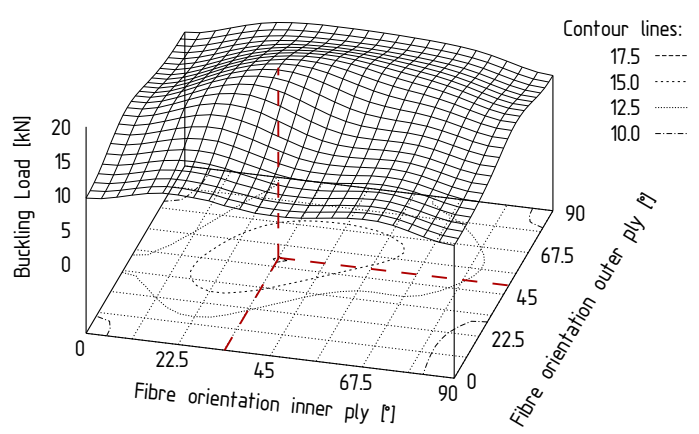

Figure 3: Buckling load of the imperfect shell structure $-\mathrm{w}_{0} / \mathrm{t}=0.2-\mathrm{n}_{\mathrm{p}}=2$ according to Friedrich $[6]$ 
Table 2: Maximum critical buckling loads for geometrical perfect and imperfect shell structures with $w_{0} / t=0.2$ according to Friedrich et. al. [6]

\begin{tabular}{|c|c|c|c|c|c|}
\hline $\mathrm{n}_{\mathrm{p}}$ & & Shell & Stacking sequence & $\begin{array}{r}\mathrm{F}_{\text {cr,perf }} \\
{[\mathrm{kN}]}\end{array}$ & $\begin{array}{r}\mathrm{F}_{\mathrm{cr}, \mathrm{imp}} \\
{[\mathrm{kN}]}\end{array}$ \\
\hline 2 & $\begin{array}{l}\text { perf. }^{*} \\
\text { imp. }^{* *}\end{array}$ & FL2 & $\begin{array}{l} \pm 15^{\circ} / \pm 26^{\circ} \\
\pm 34^{\circ} / \pm 49^{\circ}\end{array}$ & $\begin{array}{r}29.85 \\
21.66 \\
-27.45 \%\end{array}$ & $\begin{array}{r}13.70 \\
17.56 \\
+28.14 \%\end{array}$ \\
\hline 5 & $\begin{array}{l}\text { perf. }^{*} \\
\text { imp. }^{* *}\end{array}$ & $\begin{array}{l}\text { Z17 } \\
\text { FL5 }\end{array}$ & $\begin{array}{r} \pm 30^{\circ} / 90^{\circ}{ }_{2} / \pm 23^{\circ} / \pm 38^{\circ} / \pm 53^{\circ} \\
\pm 38^{\circ} / \pm 68^{\circ} / 90^{\circ}{ }_{2} / 90^{\circ}{ }_{2} / \pm 38^{\circ}\end{array}$ & $\begin{array}{r}286.74 \\
197.70 \\
-31.05 \%\end{array}$ & $\begin{array}{r}129.18 \\
139.81 \\
+8.14 \%\end{array}$ \\
\hline
\end{tabular}

*stacking sequence derived to maximize the buckling load of the perfect shell

** stacking sequence derived to maximize the buckling load of the imperfect shell

The following findings were made when evaluating Figures 2 and 3 ;

- the laminate stacking sequences maximizing the buckling load of the geometrically imperfect shell were fundamentally different to those derived when maximizing the buckling load of the perfect shell in Zimmermann [5]. This finding was in contrast to the findings made by Khot [9] where the same laminate stacking sequence of a $\left[0^{\circ}, \theta^{\circ},-\theta^{\circ}\right]$ maximized the buckling load of a geometrically perfect and imperfect shell.

- the buckling load of the geometrically perfect shells exhibited many local maxima, whereas the imperfect shells only exhibited one global maximum buckling load. In addition, the maxima for the imperfect shell was far more robust, that is less sensitive to small changes in ply angles.

Similar findings as those summarized above could be made for the shell structures studied with three to five angle plies, that is for $n_{p}=3,4,5$. The laminate stacking sequences maximizing the buckling load of the geometrically perfect and imperfect shell differed for all number of angle ply laminates. The results summarized in Table 2 indicated that the buckling load of the FL5-shell of the geometrical imperfect structure with rotational symmetric imperfections and an imperfection magnitude of $\mathrm{w}_{0} / \mathrm{t}=0.2$ was $8 \%$ higher than the corresponding buckling load of the Z17-shells. Since the shell with five angle plies maximizing the buckling load of the geometrically perfect shell, the Z17-shell is often used as reference structure, the structural performance of the Z17-shell and the FL5-shell are studied further in this paper. To this end, geometrical imperfections other than simplified rotational symmetric imperfections, which were used to identify the FL-shells, are taken into account in combination with deviations of the laminate stacking sequence of their nominal value.

\section{STOCHASTIC ANALYSES}

Geometrically nonlinear finite element computations were performed to compare the structural performance of the Z17- and FL5-shells further. In addition to geometrical imperfections, deviations of the stacking sequence lay up were taken into account, which may occur during the manufacturing process. Since neither the geometrical imperfections nor deviations of the laminate stacking sequence from the nominal stacking sequence were known, both of the influencing parameters were varied in a stochastic manner. The geometrical imperfections in a shell were idealized as non-rotational symmetric imperfections, based on Schillinger's method 
of separation [10] and adapted to laminated composite shell structures as done in [11]. To this end, based on measured imperfection patterns of composite laminated shell structures studied in a DLR-ESA study [12] and using the method of separation, stochastic imperfection patterns were defined and used to model the geometrically imperfect shell structure. The laminate stacking sequence orientations were varied in $1^{\circ}$-steps within the range $\pm 5^{\circ}$ around the nominal stacking sequence assuming a uniform distribution. The nonlinear structural response curves were determined using dynamic implicit analyses in a displacement controlled manner and the commercial software package Ansys [13]. Linear shell elements were used and 171 elements in circumferential and 63 elements in axial direction were used to model the shell structure.

The structural response of 2000 different shell structures with varying combinations of geometrical imperfections and laminate stacking sequence imperfections are studied for the Z17and FL5-shell by evaluating the resulting load displacement curves due to an implicit dynamic computation . The nonlinear buckling loads are plotted over the number of computations performed, c.f. Figure 4. For both the Z17- and FL5-shell, 2000 nonlinear analyses were performed for each shell configuration and evaluated subsequently.

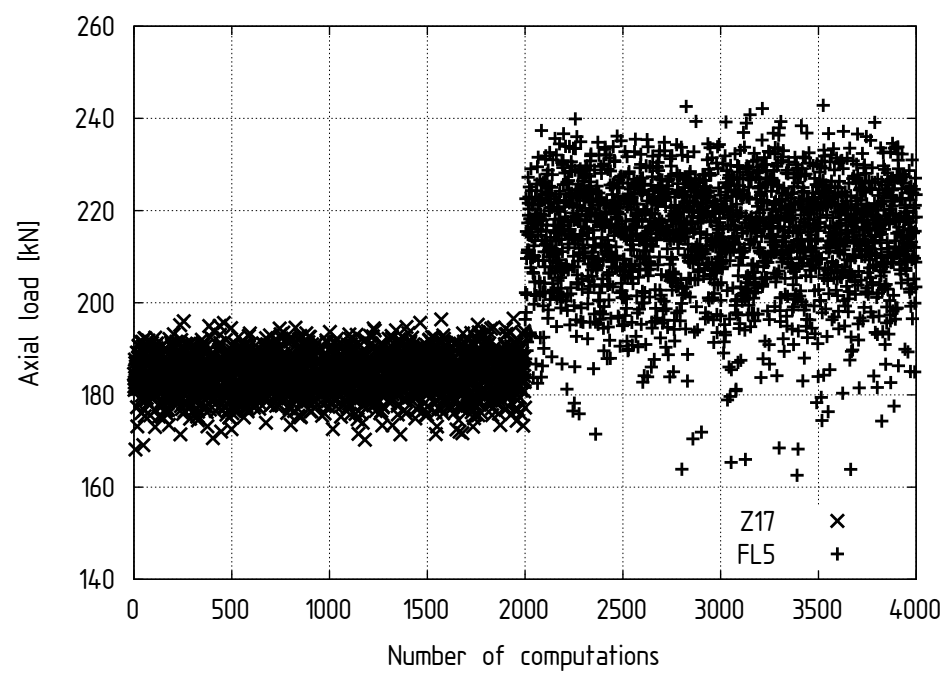

Figure 4: Stochastic studies - buckling load vs. number of computations - geometrical and lay-up imperfections

Since the scatter of the buckling loads of the Z17-shell were very low compared to the scatter observed for the FL5-shell, numerical analyses were performed using an explicit dynamic solver and the commercial software package Abaqus [14] to further evaluate the results. Because of the high resulting numerical effort, recently only 40 configurations were studied for the Z17- and FL5-shell each. The comparison of buckling loads of the Z17- and FL5-shell due to explicit dynamic and implicit dynamic analyses are summarized in Figure 5. The results given in Figure 5 are preliminary results, the resulting standard deviations and the corresponding design loads are not converged and thus in future studies additional computations need to be performed.

The mean values of the buckling loads, $\mathrm{F}_{\text {mean }}$, the standard deviations $\sigma$, the resulting buckling loads for $95.5 \%$ and $99.7 \%$ probability, $\mathrm{F}_{95.5 \%}$ and $\mathrm{F}_{99.7 \%}$, are summarized in Table 3 for the stochastic analyses performed using implicit and explicit solvers. The buckling loads $\mathrm{F}_{95.5 \%}$ and $\mathrm{F}_{99.7 \%}$ are thereby determined by reducing $\mathrm{F}_{\text {mean }}$ with two times or three times the standard deviation, $\sigma$. 


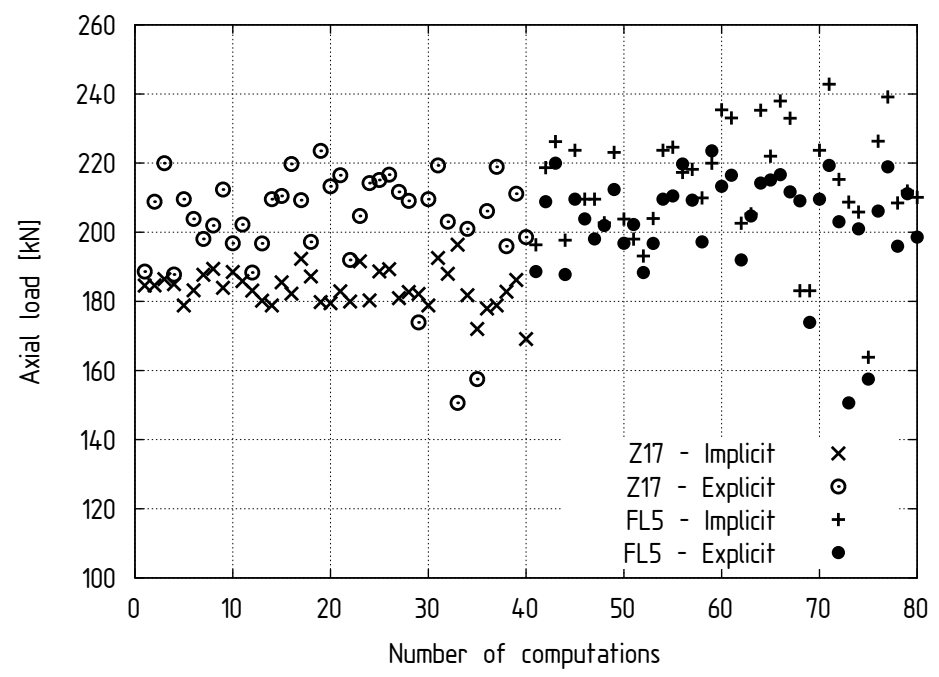

Figure 5: Stochastic studies - buckling load vs. number of computations - geometrical and lay-up imperfections explicit vs. implicit dynamic analyses using Abaqus [14] and Ansys [13]

Table 3: Evaluation of stochastic studies performed using a combination of geometric imperfections and stacking sequence lay-up imperfections

\begin{tabular}{|c|c|c|c|c|c|c|c|c|c|}
\hline \multirow{2}{*}{$\begin{array}{l}\text { Type of } \\
\text { analysis }\end{array}$} & \multirow{2}{*}{$\begin{array}{l}\text { No. of } \\
\text { comp. }\end{array}$} & \multicolumn{4}{|c|}{$\mathrm{Z} 17$} & \multicolumn{4}{|c|}{ FL5 } \\
\hline & & $\begin{array}{c}\mathrm{F}_{\text {mean }} \\
{[\mathrm{kN}]}\end{array}$ & $\begin{array}{c}\sigma \\
{[\mathrm{kN}]}\end{array}$ & $\begin{array}{c}\mathrm{F}_{95.5 \%} \\
{[\mathrm{kN}]}\end{array}$ & $\begin{array}{c}\mathrm{F}_{99.7 \%} \\
{[\mathrm{kN}]}\end{array}$ & $\begin{array}{c}\mathrm{F}_{\text {mean }} \\
{[\mathrm{kN}]}\end{array}$ & $\begin{array}{c}\sigma \\
{[\mathrm{kN}]}\end{array}$ & $\begin{array}{c}\mathrm{F}_{95.5 \%} \\
{[\mathrm{kN}]}\end{array}$ & $\begin{array}{c}\mathrm{F}_{99.7 \%} \\
{[\mathrm{kN}]}\end{array}$ \\
\hline $\begin{array}{l}\text { dynamic } \\
\text { implicit }\end{array}$ & 2000 & 184.41 & 4.47 & 175.47 & 171.00 & 214.23 & 12.12 & 189.99 & 177.87 \\
\hline $\begin{array}{l}\text { dynamic } \\
\text { explicit }\end{array}$ & 40 & 204.66 & 9.29 & 186.08 & 176.79 & 203.10 & 15.56 & 171.98 & 156.43 \\
\hline
\end{tabular}

The following findings were made when evaluating the results given in Figures 4 and 5 and those results summarized in Table 3 :

- the mean value of the FL5-shell's buckling load was higher than the mean value of the Z17-shell's buckling load for the dynamic implicit analyses

- the standard deviation of the FL5-shell's buckling load was higher than the standard deviation of the Z17-shell's buckling load that is the scatter of the FL5-shell's buckling loads was higher than for the Z17-shell

- the preliminary results due to the explicit dynamic analyses indicated that the standard deviation of the Z17-shell increased more significantly than the standard deviation of the FL5 shell when changing from implicit to explicit dynamic analyses

- the design load for a 99.7\% probability level of the FL5 shell were $4 \%$ higher than the design load for a $99.7 \%$ probability level of the Z17 shell when using implicit dynamic analyses and the commercial finite element package Ansys [13]

- the design load for a 99.7\% probability level of the FL5 shell were $11.5 \%$ lower than the design load for a $99.7 \%$ probability level of the Z17 shell when using explicit dynamic analyses and the commercial finite element package Abaqus [14] 
In Friedrich et. al. [6], it was shown that determining the nonlinear buckling load of the Z17-shell was computationally demanding. Due to the rather small bending stiffness, the shell revealed a distinct rotational symmetric pre-buckling state such that determining the nonlinear buckling load became difficult. This finding was confirmed by the studies here performed using implicit and explicit dynamic solving algorithms and using the commercial finite element software packages Abaqus [14] and Ansys [13]. Furthermore, it was shown that evaluating the structural performance of the two composite shell structures, Z17- and FL5-shells, is a demanding task, which is influenced by the actual physical behaviour and numerical difficulties when determining the structural response. The final numerical evaluation of the structural performance of the Z17- and FL5-shells could not be completed in this paper; but, additional studies are needed to allow for a final numerical evaluation.

\section{SUMMARY AND OUTLOOK}

In this paper, the structural performance of two shell structures, the Z17- and FL5-shell was studied for geometrical imperfections other than rotational symmetric imperfections and stacking sequence lay-up imperfections. Dynamic implicit and explicit analyses were performed and the results were evaluated with regard to the statistical buckling load predictions. The discrepancy between implicit dynamic and explicit dynamic results discussed above needs to be readdressed and a solving algorithm leading to robust buckling load predictions needs to be chosen for future studies. Thus, a final numerical assessment on the structural performance of the Z17- and FL5-shell may be realized.

Additionally, it would be desirable to compare the structural performance of the Z17- and FL5-shell in an experiment. To this end, these two shell structures need to be manufactured using the same manufacturing process and the same mandrel. Thus, additional insight on the structural behaviour of the Z17- and FL5-shell can be gained and serve as basis for further discussions.

\section{REFERENCES}

[1] N. J. Hoff. Perplexing Behavior of Thin Circular Cylindrical Shells in Axial Compression. AFOSR-66-0155. Department of Aeronautics and Astronautics, Stanford University, 1966.

[2] David Bushnell. "Buckling of Shells-Pitfall for Designers". In: AIAA Journal 19.9 (1981). DOI: $10.2514 / 3.60058$, URL: http://dx.doi.org/10.2514/3.60058.

[3] S. Castro et al. "Exploring the constancy of the global buckling load after a critical geometric imperfection level in thin-walled cylindrical shells for less conservative knockdown factors". In: Thin-Walled Structures 72 (2013), pp. 76-87.

[4] L. Friedrich, T.-A. Schmid-Fuertes, and K.-U. Schröder. "Comparison of theoretical approaches to account for geometrical imperfections of unstiffened isotropic thin walled cylindrical shell structures under axial compression". In: Thin-Walled Structures 92.0 (2015), pp. 1 -9. ISSN: 0263-8231. DOI: http://dx.doi.org/10.1016/j.tws. 2015.02 .019 .

[5] R. Zimmermann. "Optimierung axial gedrückter CFK-Zylinderschalen”. PhD thesis. Universität-Gesamthochschule Siegen, 1991.

[6] L. Friedrich et al. "Stacking sequence influence on imperfection sensitivity of cylindrical composite shells under axial compression”. In: Composite Structures 134 (2015), pp. 750 
-761. ISSN: 0263-8223. DOI: http://dx.doi.org/10.1016/j.compstruct. 2015.08 .132 .

[7] R. Zimmermann. "Buckling Research for imperfection Tolerant Fiber Composite Structures". In: Spacecraft Structures, Materials and Mechanical Engineering, Proceedings of the Conference held by ESA, CNES and DARA in Noordwijk, 27-29 March 1996.

[8] L. Friedrich and Hans.-G. Reimerdes. "Imperfection Sensitivity of Circular Cylindrical Shells of Varying length Subjected to Axial Compression". In: 54th AIAA Structures, Structural Dynamics, and Materials Conference. 2013. DOI: 10.2514/6.2013-177 1 .

[9] N. S. Khot and V. B. Venkayya. Effect of fiber orientation on initial postbuckling behaviour and imperfection sensitivity of composite cylindrical shells. Tech. rep. Technical Report AFFDL-TR-70-125. Air Force Flight Dynamics Laboratory, 1970.

[10] D. Schillinger and V. Papadopoulos. "Accurate estimation of evolutionary power spectra for strongly narrow-band random fields". In: Computer Methods in Applied Mechanics and Engineering 199.17-20 (2010), pp. 947 -960. Dor: http: / / dx .doi .org/10 . $1016 /$ j.cma.2009.11.008.

[11] Jendi Kepple et al. "Stochastic analysis of imperfection sensitive unstiffened composite cylinders using realistic imperfection models". In: Composite Structures 126.0 (2015), pp. 159 -173. ISSN: 0263-8223. DOI: http://dx.doi.org/10.1016/j.comps truct.2015.02.063.

[12] R. Degenhardt, L. Bethge, and L. Kärger. Probabilistics aspects of buckling knock down factors - tests and analysis, final report. Tech. rep. ESA contract 19709/06/NL/IA, 2007.

[13] Ansys - Software Package. 2015.

[14] ABAQUS 6.13 - Software Package. Dassault Systems. 2013. 